\title{
A New Research on Identify Defects in Stainless Steel Plate Using Image Processing
}

\author{
V. Elanangai, K. Vasanth
}

\begin{abstract}
Quality management is very challenging in the production of steel plates. Identifying the defects in surface of steel plates is highly challenging and plays a vital role in high percentage and in quality control process also. In most of the production fields, only manual way of checking the defects is done. Due to which the following constraints are faced. Less efficiency, increase in production cost etc.. In industries, an automated technique which provides better detection, recognition and classification as well is used. Image processing identifies the defect and classifies using MATLAB.
\end{abstract}

Keywords: Defect detection, classification, recognition, MATLAB.

\section{INTRODUCTION}

In the current scenario a method which is used to enhance an image and gives us the informative contents by processing the input image is Image processing. In Image processing an image is an input, and output will be a digital image or datas associated with that image. Image Processing system usually includes images as two dimensional one and apply some processing methods to the image. The image processing do not restrict itself to certain area which should be studied but can also be analyzed.

The Images which are generally taken for analyze is from image sensors from satellites. Processing is usually done with the help of computers to manipulation raw data to get a digital images. Since the raw data will have flaws in it, should be processed to get originality of information. The general steps involved in image processing are Preprocessing, image enhancement and display, data extraction.

In today's, scenario, automation is done for various stages of manufacturing process. In metal production fields the manual way of visualizing for defects is done. Defects are of two types, with irregularity and change in orientation . Two cases are different but concept wise same approaches. First one shows defects in structure but in some regions sudden irregularity, the second one is change in the orientation. Two methods are similar in the approach that for different kind of patterns and defects it can be applied. Manual checking needs prior knowledge. Most probably, it is not in particular only about surface defects. In any detection methods for example, detection of edges, changes occur . In production fields, failure of machine happens for various reasons. An automated method to identify the defects as an alternative can be used.

V. Elanangai, Research Scholar, Sathyabama Institute of Science and

Dr. K. Vasanth, Professor, Department of ECE, Vidya Jyothi Institute of Technology, Hyderabad, Telangana, India
Revised Manuscript Received on 14 September, 2019. Technology, Chennai, Tamilnadu, India.

\section{LITERATURE REVIEW}

The surface defects for four kinds of steel defects in the industrial applications were investigated. They are holes, scratches, coil break and rust. The experimental result gave high accuracy in the corresponding defects and the advantageous factor is the usage of 250 steel defect sample images for testing[1]. The accuracy of defect detection using methods like GLCM, Gabor wavelet, Gaussian filter segmentation method and combination of these methods give relatively less accuracy. Hence a new method implementing the Gabor filter bank was created for the reference and test images.[2]. The study on the two classes of stainless steel plates and three groups of defects formed on them was made. Based on this scale invariant feature transform (SIFT) or speed up robust features (SURF) descriptors, the classification is done based on the artificial neural networks (ANN). The results of the experiments show that the usage of class of invariance SIFT or SURF gives the high accuracy of the defects[3]. An efficient approach for detection and classification of defects on a stainless steel plate is like The defects are located and segmentation is done using the morphological operations. The GLCM attributes and geometrical attributes are extracted in order to characterize the defective region, back propagation neural network is used. The proposed system has been able to detect the defects in real time successfully[4]. A method to detect the defects on the stainless steel plates using SIFT for the defective region detection and also extracting the features. The classification method used is Scalar vector machine. The SVM can be able to generate many features for training the classifier from a few images[5]. The edge information of cold-roll steel sheets has been detected and investigated by using the digital image processing toolbox of the software MATLAB, defects detection of cold-roll steel surface based on MATLAB has a quite satisfying performance[6]. They have presented a survey for identifying the clustering algorithms which can be used for the image segmentation. In this they explained that with the clustering one can perform the image segmentation in a very efficient manner. Clustering algorithm on which they focused in this survey are from both the categories i.e. supervised and unsupervised manner. They mentioned that clustering the image segmentation process still needs some improved technology for the feature extraction because large database used cause variation in feature representation. The unsupervised algorithms are suffering from inefficiency problem due to this they require 
a serious computational efforts for image segmentation[7]. A Visual Inspection System to detect and classify rolled steel defects using methods like Computer Vision techniques and Artificial Neural Network. Defects like welding, clamp and identification hole were classified using Hough Transform[8]. In Future, the segmentation process will be done based on an effective segmentation approach for effective partitioning a defect region from other parts[9]. In this paper an approach is suggested to detect the location of defect on the surface of steel sheet by using Gabor Wavelet and variance. Values of partial images are inspected both normalized and non-normalized in the limit of $[0,1]$. Altogether, the amount of correct detection of defective regions is improved in this approach with respect to other approaches[10]. In this a surface detection algorithm for hot rolled steel plates is being developed. The algorithm integrates undecimated wavelet transform, mathematical morphology and radon transform. It determined the positions of defects by maximum modulus of inter-scale correlation coefficients at first, and then classifies the defects by prior knowledge about defects. The algorithm were tested with 563 samples. Experimental results show that the algorithm is not sensitive to scales, water marks and uneven illumination, and the recognition rate is up to $90.23 \%$ [11]. Pre-processing operations on the image such as image enhancement, noise reduction and edge detection[12]. A new segmentation of color image is proposed based on granular computing clustering algorithm. The segmentation experimental results shown that (1) GrCC segmentation is a quick segmentation compared with Kmeans and FCM segmentations, (2) GrCC segmentation is better than Kmeans and FCM from the aspects of GCE, VI, and RI. (3) Loss of entropy only is a numerical measure for the segmentation of color image without the corresponding human segmentation[14].

\section{EXISTING SYSTEM}

A best estimation algorithm is used that estimates the mean with low dependent on the values of a numerical distribution which produces a more accurate estimate of the mean. The high and low values which are outlined can be replaced with closer value to the mean, so that the estimate value is slightly moved towards the mean. The formula used for k-winsorized mean is

$$
\bar{y}_{w k}=\frac{1}{n}\left\{(k+1) y_{(k+1)}+\sum_{i=k+2}^{n-k-1} y_{(i)}+(k+1) y_{(n-k)}\right\}
$$

Consider 10 ordered samples

\section{$\mathrm{X} 1, \mathrm{X} 2, \mathrm{X} 3, \mathrm{X} 4, \mathrm{X} 5, \mathrm{X} 6, \mathrm{X} 7, \mathrm{X} 8, \mathrm{X} 9, \mathrm{X} 10$}

Where

X1- smallest, X2- Largest of the sample

$10 \%$ of winsorized sample refers to trimming of one samples on either sides

\section{$\mathrm{X} 1, \mathrm{X} 2, \mathrm{X} 3, \mathrm{X} 4, \mathrm{X} 5, \mathrm{X} 6, \mathrm{X} 7, \mathrm{X} 8, \mathrm{X} 9, \mathrm{X} 10$}

$10 \%$ of trimming refers to (10 number of samples $X 0.1$ ) $=1$ (sample to be trimmed on each side)

In order to overcome the disadvantages of K-Winsorized mean a modified version of the existing K-Winsorized mean is being proposed.

Advantage of modified K-Winsored mean is, Smaller sample sizes might compel you to use less trimming. The winsorized mean uses more information from the distribution or sample than the median. The winsorized mean of a sample is unlikely to produce an unbiased estimator for either the mean or the median. Modified Winsorized Mean or equal to median is a reasonable estimate of central tendency or mean for almost all statistical models has better resistant against outliers. Values obtained using Winsorized mean are very closer now duplicate X2 and $\mathrm{X} 9$ twice

$$
\mathrm{X} 2, \mathrm{X} 2, \mathrm{X} 3, \mathrm{X} 4, \mathrm{X} 5, \mathrm{X} 6, \mathrm{X} 7, \mathrm{X} 8, \mathrm{X} 9, \mathrm{X} 9
$$

Then Winsorized

Mean=

$(\mathrm{X} 2+\mathrm{X} 2+\mathrm{X} 3+\mathrm{X} 4+\mathrm{X} 5+\mathrm{X} 6+\mathrm{X} 7+\mathrm{X} 8+\mathrm{X} 9+\mathrm{X} 9) / 10$

\section{PROSPOSED SYSTEM}

In our proposed system we de-noise the image using the nonlinear filter called modified k-winsorized mean.

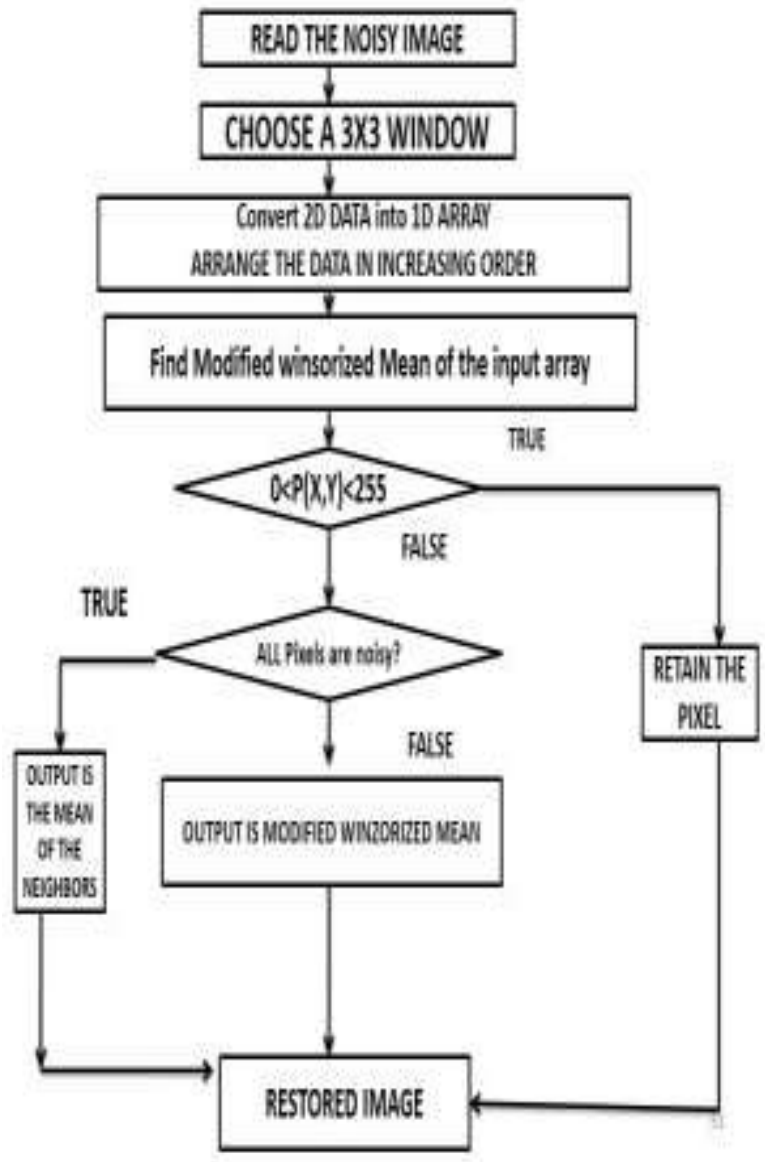

Fig 1 Flowchart of Proposed System

There are 3 different cases for the calculation of the winsorized mean. They are:

1. Processing pixel is noisy

2. All the pixels considered are noisy

3. Processing pixel is not noisy

\subsection{Case 1}

Processing pixel is noisy

Published By Blue Eyes Intelligence Engineering 


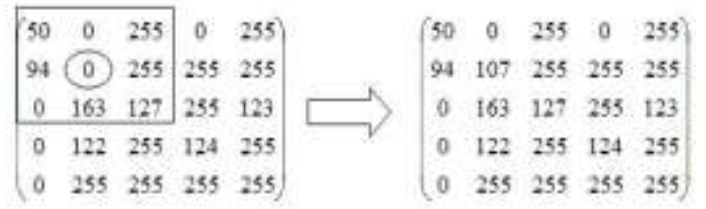

Unsorted array: $\quad 5094000163255255127$

Sorted array:

$\begin{array}{lllllll}0 & 0 & 0 & 50 & 94 & 127 & 163255\end{array}$

Trimmed Array:

5094127163

Modified Winsorized Mean:

$$
(50+50+94+127+163+163) / 6=107
$$

\subsection{Case 2}

All the pixels considered are noisy

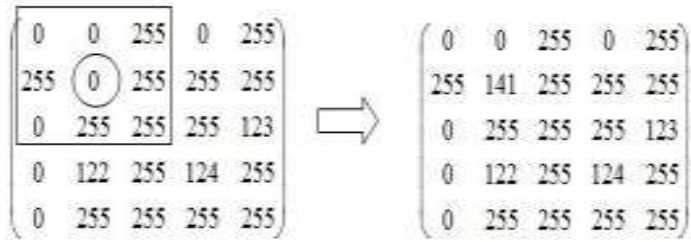

Processed pixel is noisy that is " 0 "

Output $=$ mean of neighbours

$$
\begin{array}{ll}
= & (0+255+0+0+0+255+255+255+255) / 9 \\
= & 141
\end{array}
$$

\subsection{Case 3}

Processing pixel is not noisy

$$
\left(\begin{array}{ccc|cc}
0 & 0 & 255 & 0 & 255 \\
94 & 177 & 255 & 255 & 255 \\
0 & 103 & 255 & 255 & 123 \\
0 & 122 & 255 & 124 & 255 \\
0 & 255 & 255 & 255 & 255
\end{array}\right) \longrightarrow\left(\begin{array}{ccccc}
0 & 0 & 255 & 0 & 255 \\
94 & 177 & 255 & 255 & 255 \\
0 & 103 & 255 & 255 & 123 \\
0 & 122 & 255 & 124 & 255 \\
0 & 255 & 255 & 255 & 255
\end{array}\right)
$$

The processed pixel is not noisy that is 177 . So, pixel is left unaltered.

\section{RESULTS AND DISCUSSION}

\subsection{De-Noising}

The input is exposed to salt and pepper noise as it is the noise that commonly exists during the transmission of images. Here, we use K-Winsorized mean in order to denoise the image.

Noise $=0.1 \mathrm{~dB}$
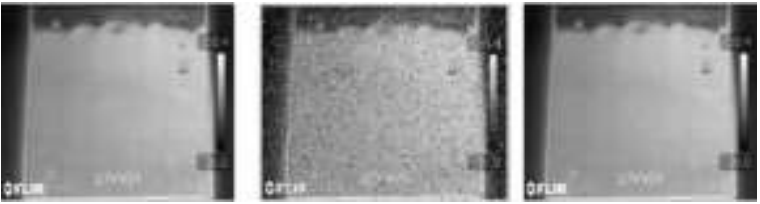

Fig. 2 Denoising process with $10 \%$ Noise
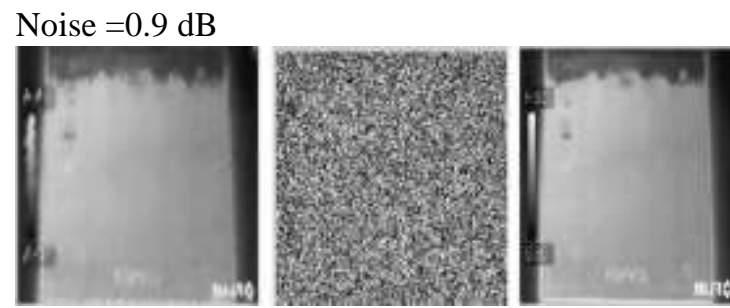

Fig.3 Denoising process with $90 \%$ Noise

\subsection{Edge Detection}

In edge detection we had compared different edge detection methods like Canny edge detector, Perwitt edge detector, Sobel edge detector, Roberts edge detector, LoG edge detector.

These edge detectors are compared with each other based on four factors:

PSNR - Peak Signal to noise Ratio

SSIM - Structural Similarity Index Metric

IEF - Image Enhancement Factor

MSE - Mean Square Error

\subsubsection{Canny edge detector}
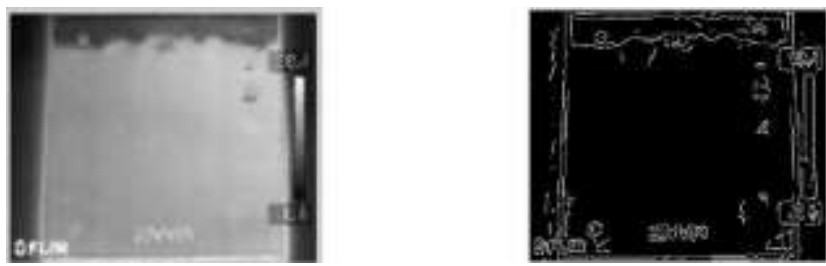

Fig 4 Grayscale Image \& Edge detection using canny

5.2.2 Sobel edge detector
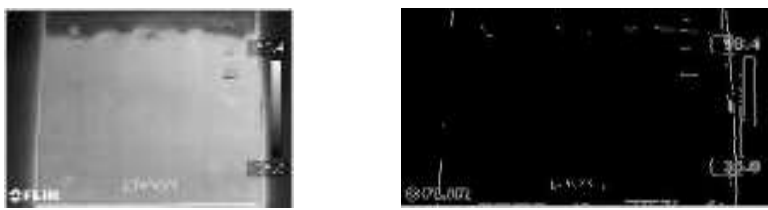

Fig 5 Grayscale Image \& Edge detection using Sobel

\subsubsection{Roberts edge detector}
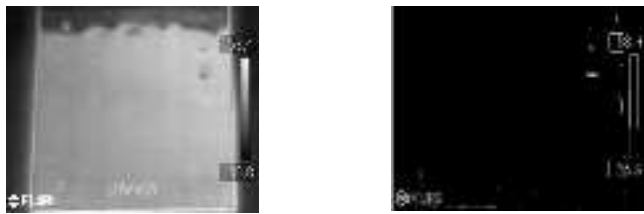

Fig 6 Grayscale Image \& Edge detection using Roberts

\subsubsection{Perwitt edge detector}
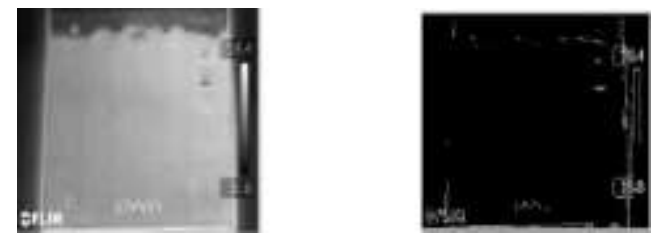

Fig 7 Grayscale Image \& Edge detection using Prewitt 

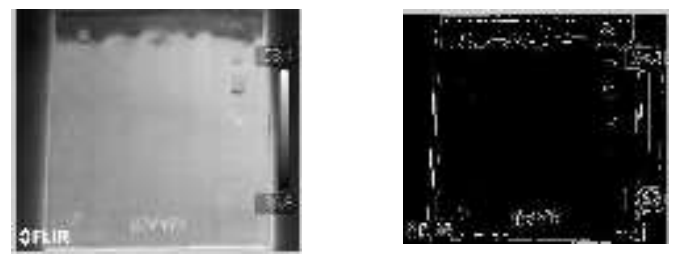

Fig 8 Grayscale Image \& Edge detection using Log

Table 1 SSIM Factor of various operators

\begin{tabular}{|l|l|l|l|l|l|}
\hline Noise Percentage & Canny & Sobel & Perwitt & Roberts & LoG \\
\hline 10 & 0.9932 & 0.9932 & 0.9933 & 0.9929 & 0.9932 \\
\hline 20 & 0.9849 & 0.9848 & 0.9858 & 0.9856 & 0.9858 \\
\hline 30 & 0.977 & 0.9763 & 0.9674 & 0.9768 & 0.9768 \\
\hline 40 & 0.9679 & 0.9666 & 0.977 & 0.9672 & 0.9673 \\
\hline 50 & 0.9551 & 0.9549 & 0.9553 & 0.9558 & 0.9561 \\
\hline 60 & 0.9407 & 0.9404 & 0.9508 & 0.9412 & 0.9405 \\
\hline 70 & 0.9216 & 0.9206 & 0.9207 & 0.9221 & 0.9226 \\
\hline 80 & 0.8894 & 0.892 & 0.894 & 0.8931 & 0.8956 \\
\hline 90 & 0.842 & 0.8418 & 0.8398 & 0.8465 & 0.8361 \\
\hline
\end{tabular}

Table 2 MSE Factor of various operators

\begin{tabular}{|l|l|l|l|l|l|}
\hline Noise Percentage & Canny & Sobel & Perwitt & Roberts & LoG \\
\hline 10 & 9.7939 & 10.9956 & 10.2118 & 11.9349 & 9.7766 \\
\hline 20 & 23.8032 & 23.425 & 21.3481 & 22.1942 & 21.905 \\
\hline 30 & 37.8556 & 37.4954 & 49.189 & 36.7545 & 37.3054 \\
\hline 40 & 47.3315 & 48.2695 & 36.6358 & 48.2744 & 50.371 \\
\hline 50 & 63.5484 & 67.0218 & 68.3177 & 66.0262 & 63.887 \\
\hline 60 & 84.2318 & 84.6887 & 86.9242 & 84.3603 & 89.1611 \\
\hline 70 & 111.2808 & 107.3757 & 112.9434 & 111.3069 & 114.7345 \\
\hline 80 & 152.6216 & 154.4616 & 152.0048 & 154.5079 & 146.689 \\
\hline 90 & 261.2941 & 261.0109 & 266.426 & 244.8254 & 255.9564 \\
\hline
\end{tabular}

Table 3 PSNR Factor of various operators

\begin{tabular}{|l|l|l|l|l|l|}
\hline Noise Percentage & Canny & Sobel & Perwitt & Roberts & LoG \\
\hline 10 & 38.2213 & 37.7186 & 38.0755 & 37.3626 & 38.2289 \\
\hline 20 & 34.3644 & 34.434 & 34.8371 & 34.6684 & 34.7234 \\
\hline 30 & 32.3495 & 32.391 & 31.2121 & 32.4777 & 32.4084 \\
\hline 40 & 31.3793 & 31.2941 & 32.4918 & 31.2938 & 31.1102 \\
\hline 50 & 30.0998 & 29.8686 & 29.7855 & 29.9336 & 30.0767 \\
\hline 60 & 28.876 & 28.8525 & 28.7394 & 28.8694 & 28.629 \\
\hline 70 & 27.6666 & 27.8217 & 27.6022 & 27.6656 & 27.5339 \\
\hline 80 & 26.2946 & 26.2146 & 26.3122 & 26.2413 & 26.4668 \\
\hline 90 & 23.9603 & 23.9642 & 23.875 & 24.2422 & 24.0491 \\
\hline
\end{tabular}

Table 4 IEF Factor of various operators

\begin{tabular}{|l|l|l|l|l|l|}
\hline Noise Percentage & Canny & Sobel & Perwitt & Roberts & LoG \\
\hline 10 & 199.9601 & 175.3903 & 191.6425 & 164.9989 & 200.9311 \\
\hline 20 & 163.5117 & 167.9164 & 178.3094 & 175.8131 & 179.0002 \\
\hline 30 & 153.7334 & 156.0429 & 155.9477 & 159.0127 & 156.3028 \\
\hline 40 & 164.7108 & 161.0205 & 158.8174 & 162.3188 & 154.1915 \\
\hline 50 & 152.7306 & 144.1673 & 143.2159 & 147.2998 & 152.4754 \\
\hline 60 & 137.6454 & 136.4958 & 134.6727 & 138.2466 & 130.3732 \\
\hline 70 & 121.8251 & 127.7853 & 119.791 & 121.8201 & 118.4062 \\
\hline 80 & 102.703 & 99.9698 & 102.2441 & 100.5764 & 106.298 \\
\hline 90 & 67.2249 & 66.5292 & 65.8633 & 71.7173 & 68.2365 \\
\hline
\end{tabular}




\subsection{Morphological Processing}

By using the canny detector the edge detection is being performed. Then the image segmentation is performed by morphological processing as it detects both internal and external boundaries.
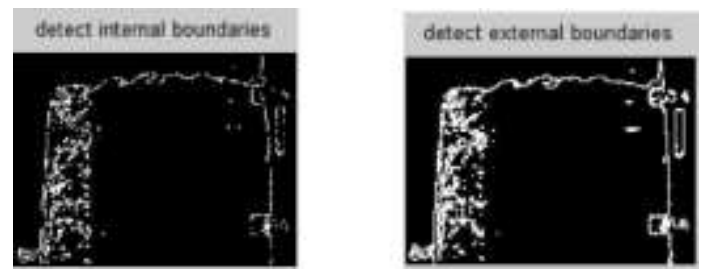

Fig 9 Internal Boundaries \& External Boundaries

\subsection{Feature Extraction}

The $\mathrm{mBm}$ is being used in order to extract the required features in the given input image.

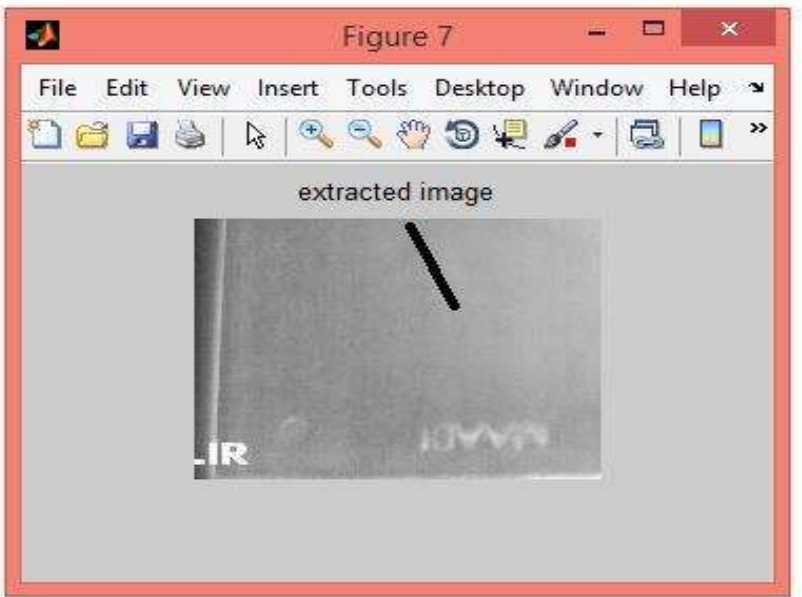

Fig 10 Feature Extraction with defective image

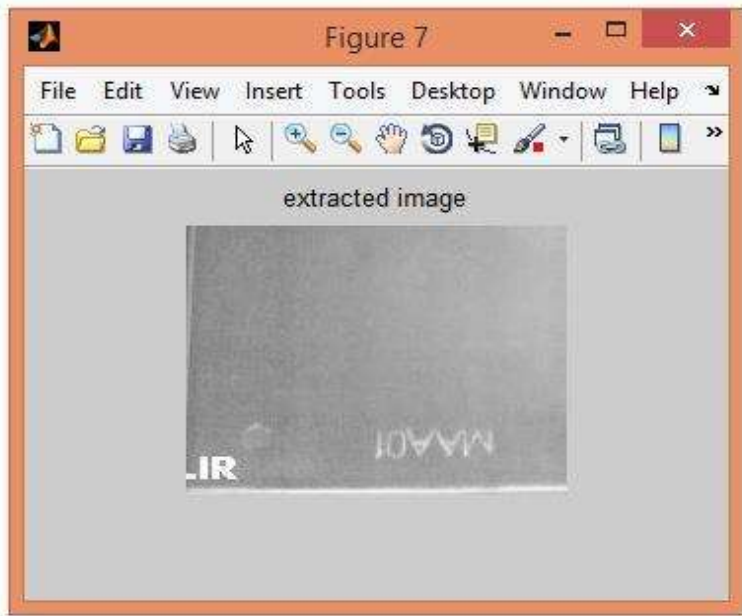

Fig 11 Feature Extraction with defect free image

5.5 Classification And GUI

Even the minor defects can be detected by using the correlation process
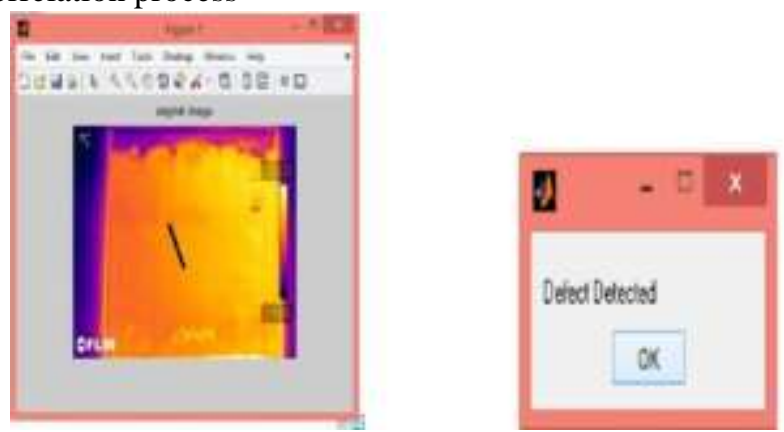

Fig 12 Defective Image \& Output Image
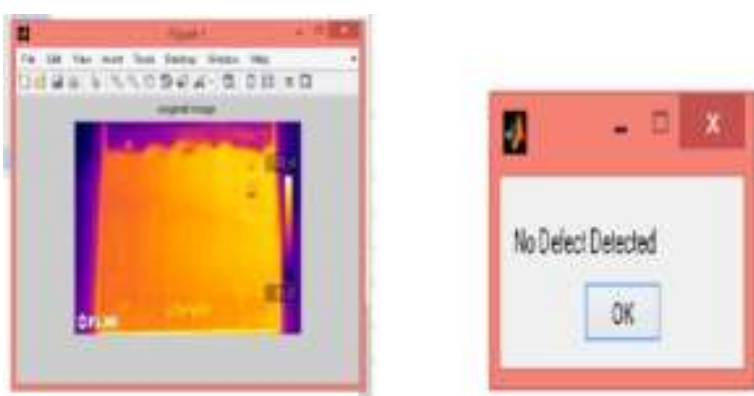

Fig 13 Defect Free Image \& Output Image 
Table 5 Comparisons of various image inputs

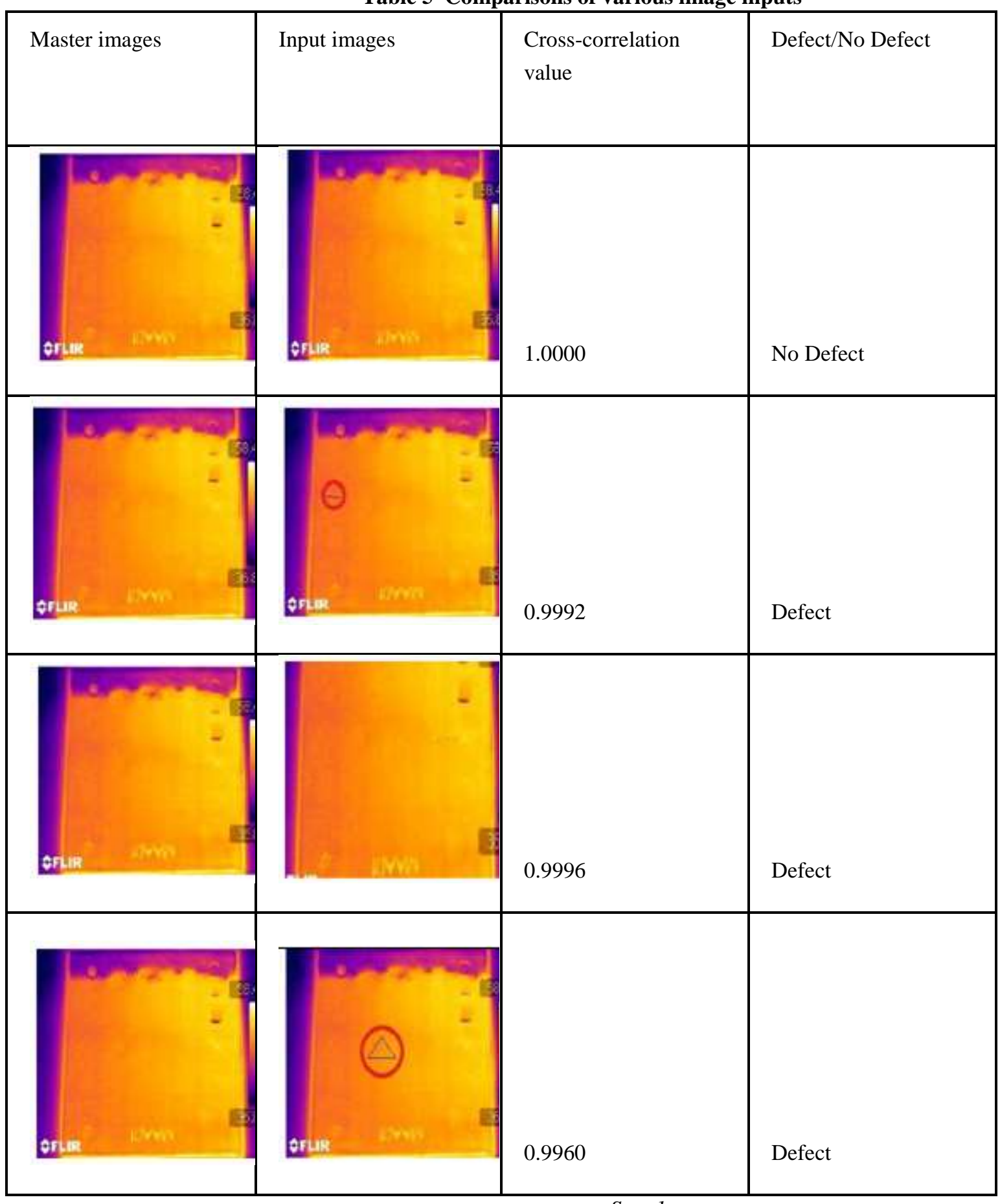

5.5.1 GUI Performance

Step 1 
International Journal of Innovative Technology and Exploring Engineering (IJITEE) ISSN: 2278-3075, Volume-8 Issue-12S2, October 2019

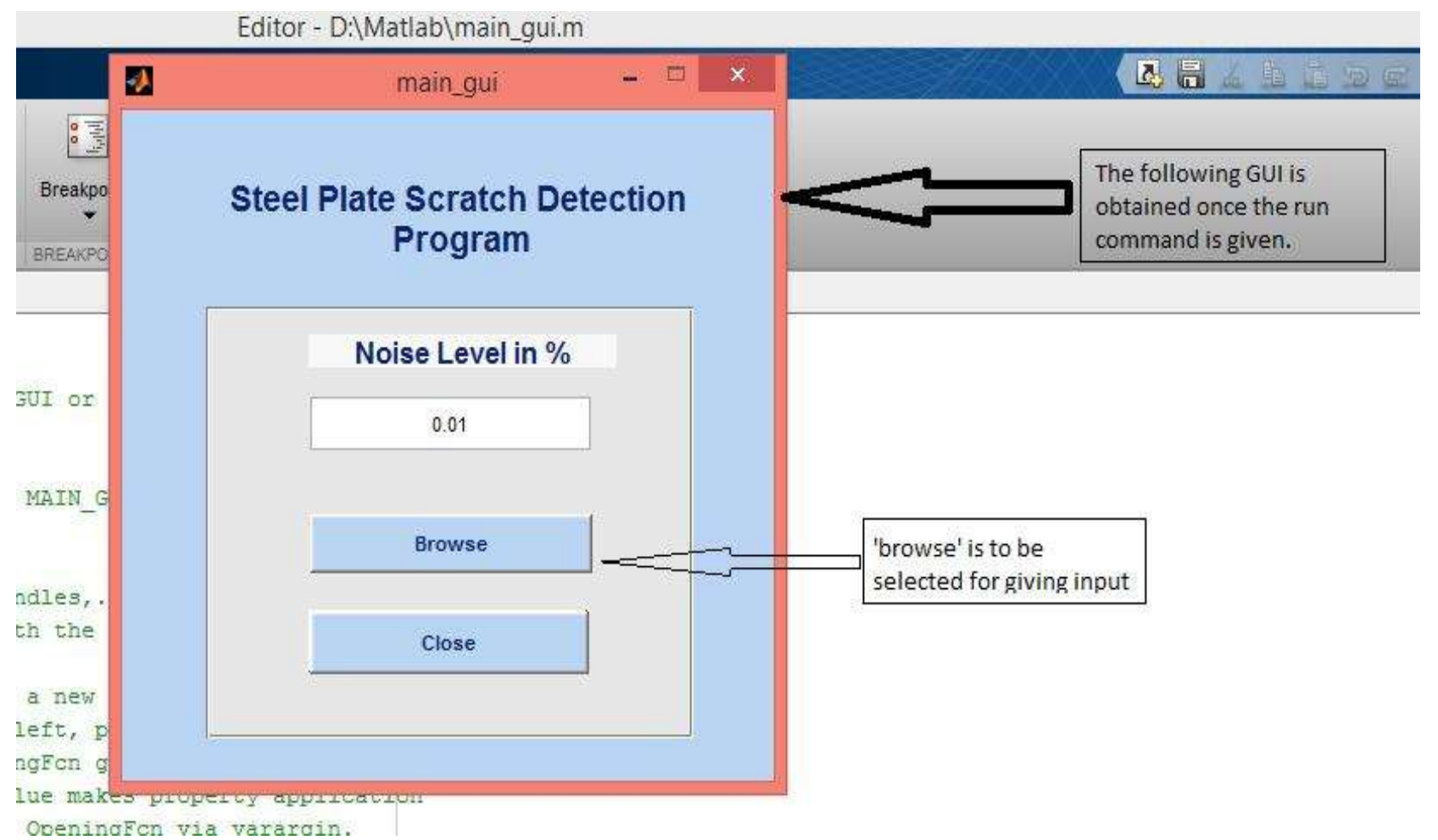

Fig14 GUI Step 1

Step 2

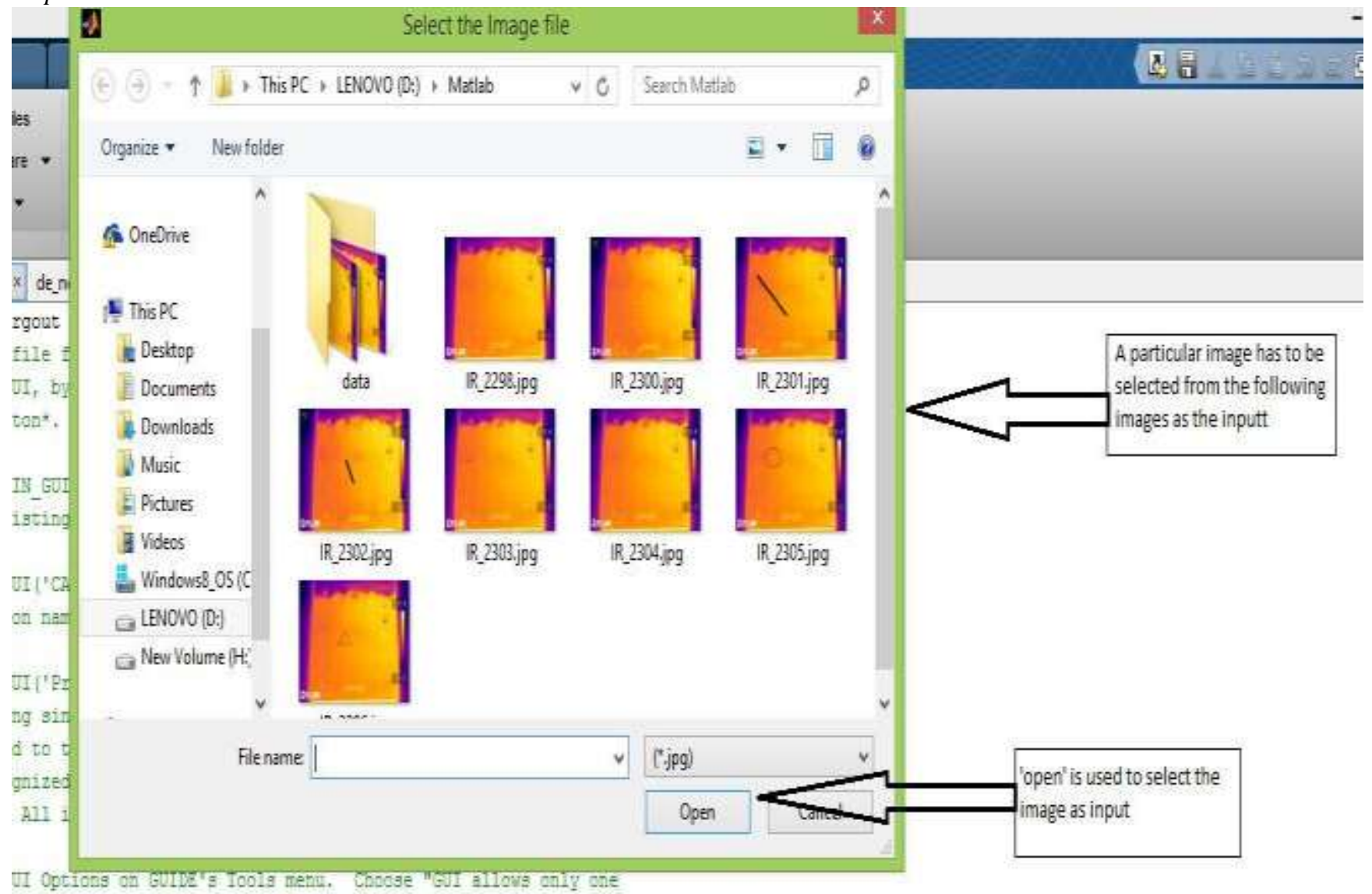

Fig15 GUI Step2

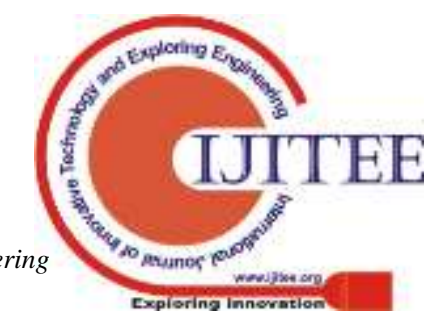


Step 3

The following outputs are being obtained

$$
\text { Figure } 1 \text { a }
$$

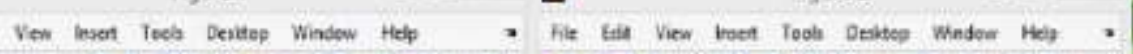

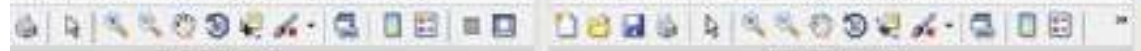

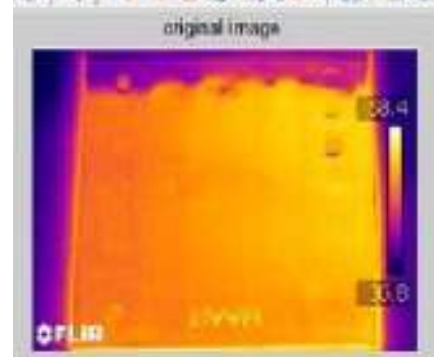
pay stake imags

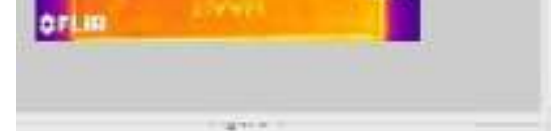

Edit Wew iven Teas Desides Window Hetp

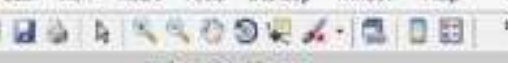
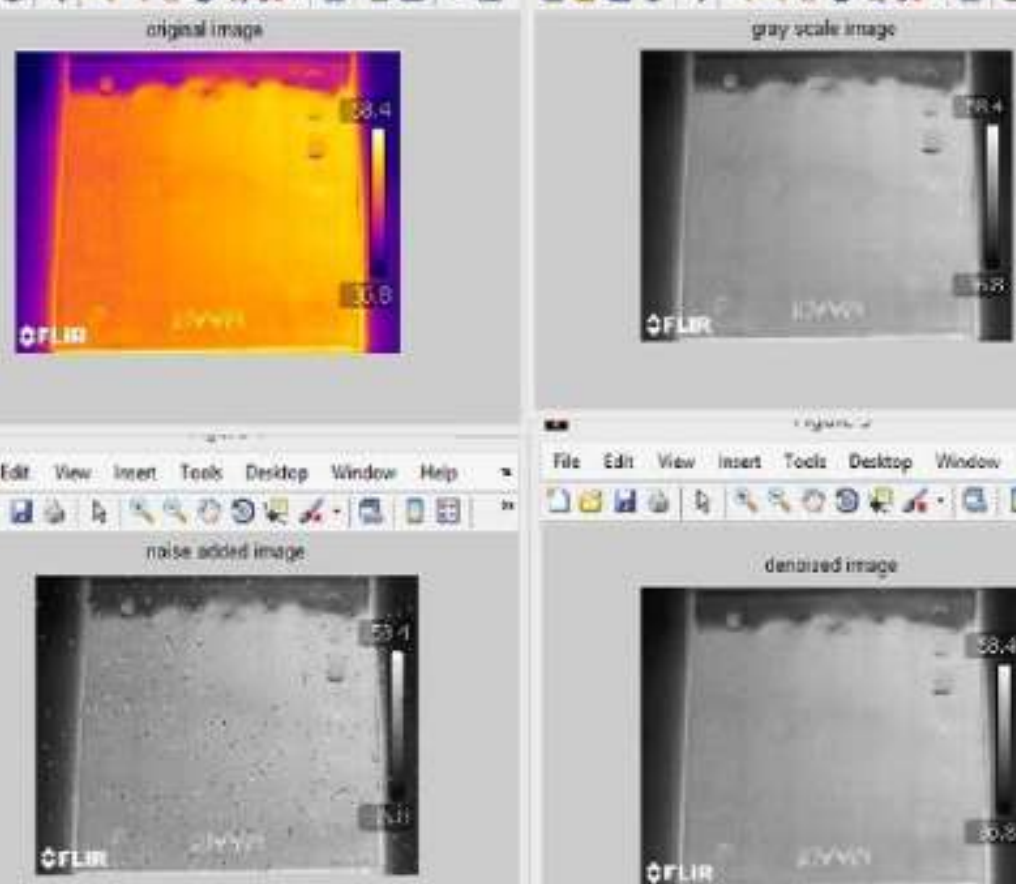

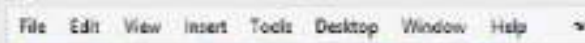
J日

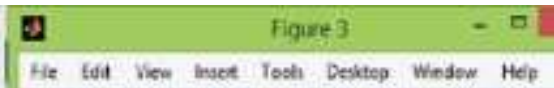

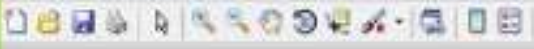
canny edege detedied GSt
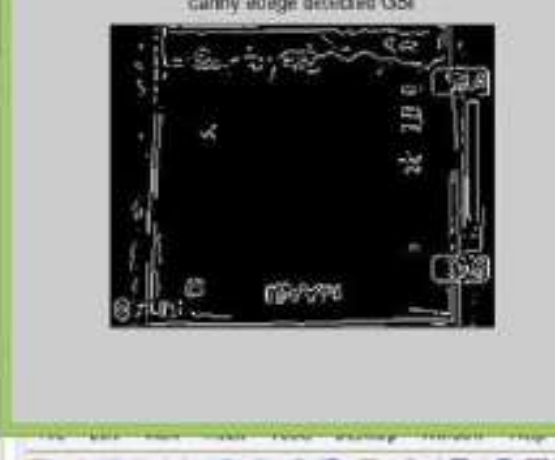

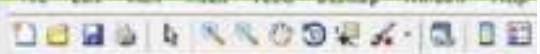
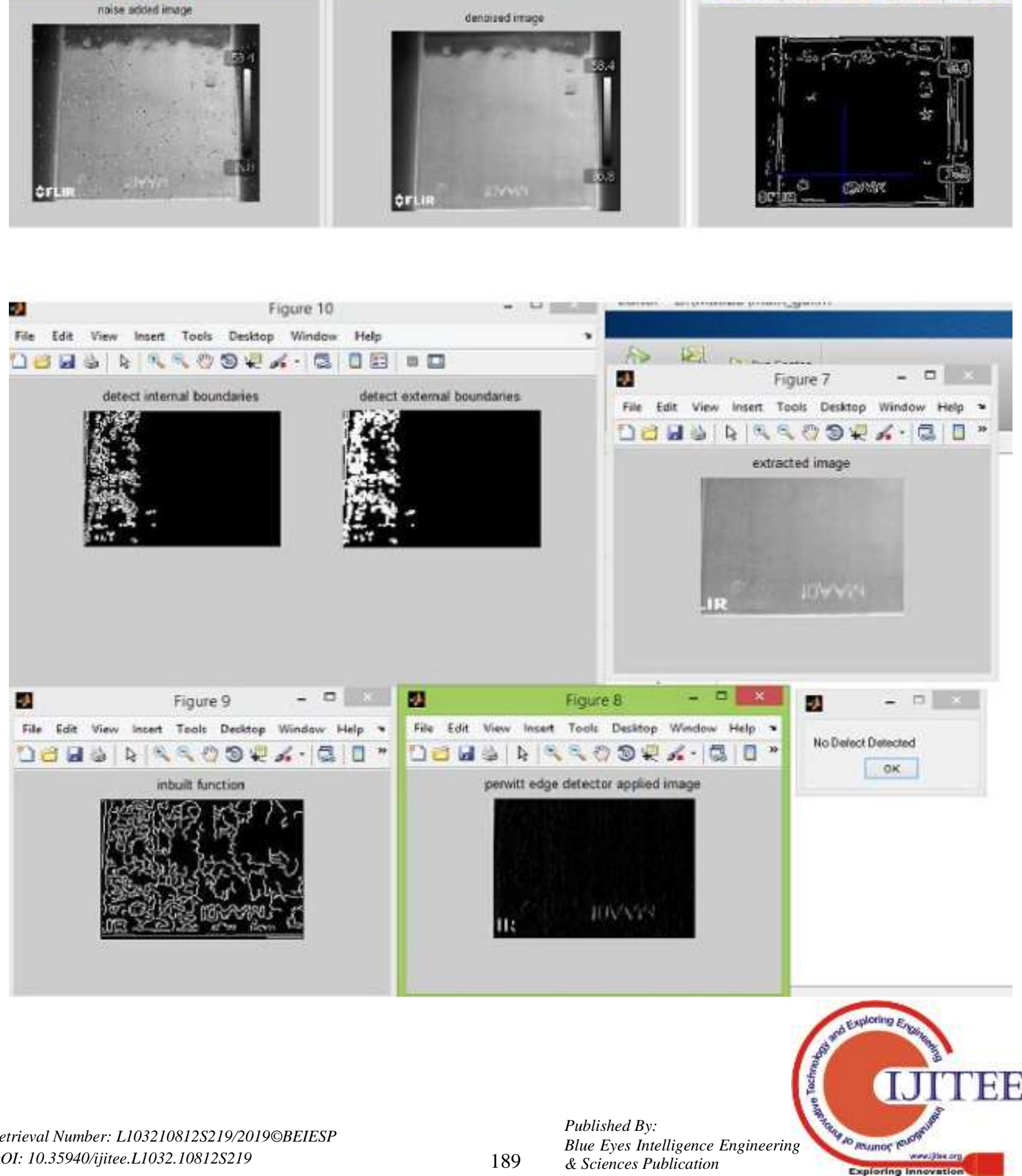


\section{CONCLUSION}

The de-noising capacity of modified K-Winsorized mean has shown very good results. Its de-noising capability has been tested at various levels of noise which range from 0.01 $(1 \%)$ to $0.999(99.9 \%)$ of noise. At each level the noise has been removed providing the accurate output. Edge detection has tested using various filters like sobel, perwitt, Roberts, canny, LoG. In order to compare the output of these filters some parameters like PSNR(Peak Signal To Noise Ratio), MSE(Mean Square Error), IEF(Image Enhancement Factor), SSIM(Structural Symmetry Indication Matrix) are considered. Comparing these parameters for all the filters as shown in tables $1,2,3,4$ it can be inferred that canny provides the best output for the edge detection. Then internal and external edges for the images are deduced using morphological processing. Multiple Brownian motion iterates the image multiple time and gets the required extracted region. A data base is developed in order to compare the images during the correlation process for classification and GUI is developed for easy usage of the user.

\section{FUTURE WORK}

The de-noising of the image lacks in its performance when the noise value is 1 which has to be improved. In Brownian motion during resizing the image to $256 * 256$ it makes delay of about 30 seconds for it. This delay time during the resizing has to be decreased or the image should be resized to $128 * 128$ in order to decrease the time lag. A database is being created for the correlation process.

The major drawback is that it can't be used for a bulk of images at a time. So, a method to improve this and make an access has to be developed. This user to classify many images at the same time.

\section{REFERENCES}

1. Rao, B. P. C., K. Nandakumar, T. Jayakumar, P. Kalyanasundaram, and Baldev Raj. "AN IMAGE PROCESSING APPROACH FOR AUTOMATED DETECTION OF DEFECTS IN STAINLESS STEEL PLATES." Image Analysis in Materials and Life Sciences: Proceedings of SCIAMAL-99, November 7-10, 1999, Kalpakkam, India(2001): 148.

2. Unay, Devrim, and Bernard Gosselin. "Apple defect segmentation by artificial neural networks." In Proceedings of BeNeLux Conference on Artificial Intelligence. 2006.

3. Elbehiery, H., A. Hefnawy, and M. Elewa. "Surface Defects Detection for Ceramic Tiles Using Image Processing and Morphological Techniques." In WEC (5), pp. 158-162. 2005.

4. Wu, Xiu-yong, Ke Xu, and Jin-wu Xu. "Application of undecimated wavelet transform to surface defect detection of hot rolled steel plates." In Image and Signal Processing, 2008. CISP'08. Congress on, vol. 4, pp. 528532. IEEE, 2008.

5. Li, Xiaohao, Ying Huang, and Qunchao Zhao. "Recognition and extraction algorithm design for defect characteristics of armor-plate flaw detection image." In Information and Computing (ICIC), 2010 Third International Conference on, vol. 4, pp. 288-291. IEEE, 2010.

6. Po-Han Chen, Heng-Kuang Shen, Luh-Maan Chang, "A
Rust Intensity Recognition Approach For Steel Bridge Surface Coating Images Based On Artificial Neural Networks". In 2011 Proceedings of the 28th ISARC, Seoul, Korea. Pages 996-1001

7. Dhanalakshmi, S., and Dr T. Ravichandran. "A new method for image segmentation." International Journal of Advanced Research in Computer Science and Software Engineering 2, no. 9 (2012).

8. Kaur, Navneet, and Mandeep Dalal. "Application of machine vision techniques in textile (fabric) quality analysis." IOSR Journal of Engineering 2, no. 4 (2012): 582-584.

9. Anand H.Kulkarn, Sheetal B.Patil, "Automated Garment identification and defect detection model based on Texture Features and PNN". In International Journal of Latest Trends in Engineering and Technology (IJLTET).

10. Landstrom, Anders, and Matthew J. Thurley. "Morphology-based crack detection for steel slabs." IEEE Journal of selected topics in signal processing 6, no. 7 (2012): 866-875.

11. Sadeghi, Mostafa, and Faezeh Memarzadehzavareh. "Flaws detection in steel plates Using Gabor Wavelet." Life Science Journal 10, no. 2s (2013).

12. Elanangai, V., Babu, V.K. "Automated system for defect identification and character recognition using IR images of SS-plates". International Journal of Recent Technology and Engineering. Volume-8 Issue-3, September 2019.

13. Akbar, Habibullah, Nanna Suryana, and Fikri Akbar. "Surface Defect Detection and Classification Based on Statistical Filter and Decision Tree." International Journal of Computer Theory and Engineering 5, no. 5 (2013): 774.

14. Martins, Luis Miguel Morais. "Automatic Visual Inspection in Industrial Problems."

15. Vasanth, K., V. Elanangai, S. Saravanan, and G. Nagarajan. "FSM-Based VLSI Architecture for the $3 \times 3$ Window-Based DBUTMPF Algorithm." In Proceedings of the International Conference on Soft Computing Systems, pp. 235-247. Springer, New Delhi, 2016.

16. K. Vasanth, Tena J, Elanangai. V, Amuthan, " Cascaded Algorithm for the removal of impulse noise variants and artifacts in images", International Journal of Applied Engineering Reasearch (IJAER), Vol 9, No. 19 (2014), pp. 5779-5796. ISSN No. 0973-4562.

17. Vasanth, K., V. Jawahar Senthil Kumar, and V. Elanangai. "Unsymmetrical Trimmed Midpoint as Detector for Salt and Pepper Noise Removal." In Advances in Computing and Information Technology, pp. 813-822. Springer, Berlin, Heidelberg, 2013. 